\title{
Is the process of stabilization of carotid plaque more dynamic than expected? a high-resolution 3D-CMR statin-naive human study
}

\author{
Robert WW Biederman 1*, David R Neff', Saundra B Grant', Ronald B Williams', Geetha Rayarao', June A Yamrozik', \\ George Angheloiu', Sobhan Kodali', Vikas K Rathi², Mark Doyle ${ }^{1}$
}

From 2011 SCMR/Euro CMR Joint Scientific Sessions

Nice, France. 3-6 February 2011

\section{Introduction}

Atherosclerosis is a dynamic process thought to stabilize with statin therapy. However, the uniformity of plaque stabilization and subsequent regression when examined under high-resolution $3 \mathrm{D} \mathrm{CMR}$ is unknown. We hypothesize that plaque characteristics illustrate marked heterogeneity with both plaque regression and progression as lipid lowering therapy is instituted.

\section{Purpose \\ Methods}

Via CMR (1.5T GE), 707-two mm contiguous in vivo slices of advanced carotid disease ( $>50 \%$; mean $64 \pm 21$ ) representing 42 complete bilateral human plaques (age $68 \pm 15 \mathrm{yrs}$ ) were analyzed for $2 \mathrm{D} / 3 \mathrm{D}$ extent of vascular wall: lipid pool, fibrous cap, outer wall area (OWA), vessel wall area (VWA), lumen area (LA) and lipid pool (LP). All were related to fasting lipids relative to \%stenosis via QPlaque (Medis). Plaque morphology was determined by CMR (T1/T2/PD) at baseline and one year following lipid lowering agent (simvastatin or simva/ ezetimibe). Plaque progression was defined as LP pre/LP post $<1$ while plaque regression was defined as LP pre/ LP post $>1$.

\section{Results}

$39 / 42$ in vivo plaques in statin naïve pts were successfully imaged. Resolution: $1 \times 1 \times 2 \mathrm{~mm}$. Pre therapy, $\mathrm{mg} / \mathrm{dL}$ range of LDLC was 60-189 (mean 142), HDLC: 23-71 and TG: $80-214$. LP represented $30 \pm 4 \%$ and fibrous laque $9 \pm 22 \%$ of total vessel wall. Post therapy, LDL was

${ }^{1}$ Allegheny General Hospital, Pittsburgh, PA, USA

Full list of author information is available at the end of the article
$66 \pm 31 \mathrm{mg} / \mathrm{dL}$. In 707 slices, 378 (53.5\%) demonstrated progression while 329 (46.5\%) showed regression. In those plaques that regressed there was significant decrease in both OWA, VWA and fibrous plaque as well as a paradoxical decrease in LA ( $<<0.0001$ for all) while he converse was true for progression $(\mathrm{p}<0.0001$ for all but LA). Specifically, LA decreased from 27.0 to $21.8 \mathrm{~mm}^{2}$ to $(19 \% ; \mathrm{p}<0.0001)$ while LA increased from 24.4 to $26.0 \mathrm{~mm}^{2}$ ( $9 \%$; $\left.\mathrm{p}=\mathrm{NS}\right)$. Segmenting for quartiles of LDL favorably trended with $\triangle \mathrm{LA} ; \mathrm{r}=0.35, \mathrm{p}=0.08$ while lumen size was related to $\Delta \mathrm{LDL}, \mathrm{p}<0.02$ ).

\section{Conclusions}

In statin naïve pts, administration of lipid modulating agents appear to have initial paradoxical effects on lumen size as assessed by high-resolution 3D CMR: as VWA and LP decreases so does the LA. Similarly, as VWA and LP increases so does LA. All appears driven by the effectiveness of the $\triangle \mathrm{LDL}$ achieved. Thus, marked heterogeneity in plaque compositional changes exist that are only resolved once $\Delta \mathrm{LDL}$ is known.

\section{Author details \\ 'Allegheny General Hospital, Pittsburgh, PA, USA. ${ }^{2}$ Bon Secours Heart and Vascular Institute, Richmond, VA, USA.}

Published: 2 February 2011

\section{doi:10.1186/1532-429X-13-S1-096}

Cite this article as: Biederman et al:. Is the process of stabilization of carotid plaque more dynamic than expected? a high-resolution 3D-CMR statin-naive human study. Journal of Cardiovascular Magnetic Resonance 2011 13(Suppl 1):096.

\section{C)

(c) 2011 Biederman et al; licensee BioMed Central Ltd. This is an open access article distributed under the terms of the Creative Commons Attribution License (http://creativecommons.org/licenses/by/2.0), which permits unrestricted use, distribution, and reproduction in any medium, provided the original work is properly cited. 